\title{
Labyrinthe
}

37 | 2011 (2)

Des séries et des vies

\section{Un sens de la réserve}

\section{Laurent Dubreuil}

\section{OpenEdition}

\section{Journals}

Édition électronique

URL : http://journals.openedition.org/labyrinthe/4194

DOI : $10.4000 /$ labyrinthe.4194

ISSN : 1950-6031

Éditeur

Hermann

Édition imprimée

Date de publication : 15 août 2011

Pagination : 67-72

ISBN : 9782705681470

\section{Référence électronique}

Laurent Dubreuil, « Un sens de la réserve », Labyrinthe [En ligne], 37 | 2011 (2), mis en ligne le 01 août

2013, consulté le 20 avril 2019. URL : http://journals.openedition.org/labyrinthe/4194 ; DOI : 10.4000/ labyrinthe.4194

Propriété intellectuelle 


\section{Un sens de la réserve}

Laurent Dubreuil

Je lis beaucoup de romans américains récents lorsque je vais à Miami Beach. Dans cet endroit où à peu près tout l'art est plastique - architecture, mode, design, peinture, sculpture, photographie, body building -, où la multiplicité des langues semble d'avance limiter la possibilité du dialogue, mon intérêt déplacé pour la textualité joue sans doute le rôle d'une compensation, que je rechercherais. Alors que j'aime South Beach pour les cruelles limitations de sa splendeur, je refilerais en contrebande (l'île de la Tortue n'est pas si loin) un peu du pouvoir des mots. Sauf qu'en lisant ce qui s'écrit prosaïquement, je suis autant frappé du creux heuristique dans les récits que je découvre. Selon une certaine filiation, les écrivains de chaque génération semblent construire des personnages à la vie de plus en plus vide. Pour en rester à une séquence américaine, John Cheever ou Richard Yates rendaient sensible la spirituelle kénose de l'existence suburbaine; consommation, frustration, dépression. Bret Easton Ellis décrit choses et personnes par leurs marques, leur prix. Le monde mental de ses protagonistes se fait de réflexes pulsionnels, d'envies de, de réactions contre. Le subjectif est objectal. Dans son récent roman précisément intitulé Richard Yates, Lin Tao répète ad libitum le nom de ses héros, comme s'il fallait toujours marteler l'index référentiel afin de maintenir un semblant d'âme, puisque ni « Dakota Fanning » ni « Haley Joel Osment » ne sont autres que les porteurs de microscopiques affects délivrés par la rencontre hasardeuse entre les circonstances et la pulsion de communiquer par Gmail. Au fond (le fond, s'agit-il bien de cela?), je n'aime guère ces romans. J'y reconnais hélas de la création littéraire, qui, je l'ai souvent écrit, répond aux autres mots, discours et jargons; avec ici une réponse essentiellement donnée à la parlure médiatique, au babil de l'ordinaire, à l'organisation langagière de l'âge technologique triomphant — plutôt qu'à la philosophie, la mystique ou la tradition poétique. L'indiscret sentiment de trouver une validation de mes théories dans ce relatif inattendu ne suffirait pourtant pas à me faire lire ces ouvrages. L'intérêt que j'ai pour eux, malgré mon manque de goût, tiendrait plutôt à ce contenu de vérité que je leur attribue, à tort 
peut-être. Après tout, je pense (depuis aussi longtemps que j'y songe) que les œuvres littéraires signifient, qu'elles nous permettent de penser plus loin, qu'elles nous aident à ressaisir ce dont elles parlent. Ma réticence au dogme d'un formalisme asémantique de l'art me pousserait alors à prendre ces romans pour la mise en œuvre d'un événement psychologique, aujourd'hui crucial, soit la domination sociale de tous ces mini-moi en série, à l'intériorité quasi extérieure. Je repose ces récits, je vais à la plage, vers Lincoln Road, ou dans un parc, et tout paraît logique ou cohérent: les automates que discernait le dubitatif Descartes depuis sa fenêtre, c'est la plupart des humains.

Voilà ce qui s'insinue dans mon cerveau, et je m'en veux. Dans un même paragraphe - celui du dessus, par exemple -, je peux donner l'impression de requérir contre la plastique au profit du textuel, de prendre le parti de la profondeur contre la surface', de séparer l'illusion du réel. Sans avoir la capacité de tout nier exactement, je ne crois pas que cette dernière phrase soit fidèle à ce que j'essaie de formuler. Cependant, je ne le puis celer, autant la phraséologie situationniste du spectaculaire et de la vie m'irrite, autant je ne me reconnais pas ou plus dans l'univoque promotion de l'extension superficielle, qui voulait se défaire de la célébration ambiguë des profondeurs. Le terme d'intériorité n'est peut-être pas adéquat, s'il consiste à ramener la vie mentale à « notre tréfonds ", et à dévaloriser nos actions, nos larmes, nos baisers, nos poèmes à des signes de profondeur. Non, il n'y a aucune raison de mépriser ce que nous partageons au nom d'une supériorité a priori de l'inaccessible intérieur. En revanche, je plaide en faveur d'une certaine réserve, qui se manifesterait $e t$ résisterait à sa conversion en manifestation. C'est le problème. Dès lors que j'existe en dehors de moi, qu'advient-il de moi? La solution substantielle classique est d'en revenir à une base, un ego, un subjectivité, une personne. Quand j'ai développé la notion de singularité, je cherchais à dire qu'en dépit de toute l'implosion de l'intenable soi, demeure la difficile possibilité d'un je qui ne sait ce qu'il est, ni s'il est vraiment, et qui néanmoins insiste, s'entête. Et qu'il vaut mieux penser ce je que d'en rester aux forteresses du sujet, ou d'épouser quelque forme radicale de behaviourisme. La réserve est un autre nom pour ce qui permet la singularité, à savoir la résistance du manifesté à sa manifestation. Ce

1. Je me rappelle que mon premier article s'intitulait « Une pleine superficie » (Au Hasard Balthazar, $\left.n^{\circ} 1,1995\right)$, qu'il traitait de David Lynch et Carl Dreyer, mais je n'en sais pas plus, et je n'irai pas vérifier. 


\section{Un sens de la réserve}

discord accrédite l'idée du rapport de conséquence entre l'intérieur et l'extérieur, tandis qu'il s'agit en fait d'un système plus complexe, où la sédimentation a lieu dans la suite de l'expression, et comme le refus de sa valeur intégrale. L'opacité non régulée de l'énoncé verbal, le retard à soi de l'œuvre de l'art témoignent également d'une telle réserve, où nous pouvons nous lover, et indépendamment de la vie-intérieure des créateurs. Maintenant, l'inquiétude serait que la résistance tombe énormément, et que la confusion entre manifesté et manifestation se justifie dans l'ensemble. Les "mini-moi en série » désigneraient un mode de production de l'humain à très faible réserve. Quel que soit l'invariant de l'inconstructible anthropogénie, il y a fort à parier que certaines sociétés, plus que d'autres, encouragent la réserve, ou l'embrigadent, la neutralisent, la restreignent. Qui sait si Miami Beach, par l'un de ses aspects du moins, ne correspond à l'image publicitaire d'une fabrique moderne, ou dite postmoderne, réputée américaine, des individus de peu de résistance? La circuiterie pulsionnelle reste et fonctionne, ramenée à ses simples mécanismes; la projection ne cache pas le projet, ni ne le déporte, ni ne l'influence, elle tend juste à l'annihiler. Comme dans The Insiders, Ellis a souvent besoin d'insérer des révélations brutales dans ses narrations étales: ce sont en fait des vampires, ou des extraterrestres. Interprétant le même type de réel, le réalisateur Gregg Araki recourt à des moyens comparables (l'alien de Nowhere, la brigade néo-nazie de The Doom Generation). J'y vois une volonté, mi-ironique mi-désespérée, de rétablir une surnaturelle réserve. Sauf que celle-ci est surtout l'ajout d'une manifestation différente, qui se donne pour une extériorité contingente. Il resterait chez Araki ou Ellis l'angoisse d'une réserve réduite au minimum vital. La dimension compensatoire, dont je parlais d'abord, est chez eux la représentation du rabattage de l'irreprésenté, avec l'irruption d'un hors-champ inattendu en guise de résistance. - Mais s'il faut faire l'hypothèse que les sociétés organisent ces croissantes formes de vie mentale miniature, que je vois à Miami et ailleurs dans mon séjour aux États-Unis et sur la Terre, j'ai mille motifs pour incriminer un certain usage de la télévision, et ce qu'il a configuré pour longtemps dans la subséquente informatisation des comportements. Il ne suffit pas de récuser la télévision en bloc pour sa structure anti-démocratique, spectaculaire, abêtissante. En célébrer la géniale postmodernité dans ses recoins les plus minables n'est pas mieux. Séparer, même, l'œuvre de certaines séries de l'immensité où elle se déploie, ainsi que le tente ce dossier, mérite 
à mon sens la considération supplémentaire de la victoire historique de « la» Télévision, qui, à ses enfants, finit par imposer comme normale une forme existentielle d'extrême réduction.

L'ouvrage fondateur de Raymond Williams, Television, est né d'une expérience personnelle que l'auteur rapporte, cette nuit qui le mit en face de l'offre abondante de programmes sur de multiples chaînes. Sous le coup de cette révélation d'une manifestation insensée, désorganisée, infinie et apparemment auto-suffisante, Williams déploie son concept de flux ou de flot (flow), pour expliquer un fonctionnement de la télévision permanente. Il est clair que, via les DVD et le streaming, l'accession nouvelle et sélective aux séries télé, soudain défalquée de la publicité et du reste de la chaîne, en interrompant le flot, permet de retarder la caducité du medium télévisuel, et en désassemble la logique principale. Par-delà les qualités de quelques séries contemporaines hors du commun (mais il y eut toujours, à un moment donné, d'assez comparables exceptions ${ }^{2}$ ), l'engouement actuel dépend largement de cette perte du flux, qui met plus aisément en relief les réussites isolées, aux yeux d'une frange de spectateurs du moins. Le flow de Williams contient davantage. Il signale aussi un procédé télévisuel consistant à privilégier une occupation constante, frénétique (penser au montage " clippé », qui s'est largement imposé dans d'immenses segments du filmé3), qui vise à faire de la manifestation son début, son « milieu », sa fin. Dois-je préciser que l'épisode marquant pour la réflexion de Williams eut lieu un soir à Miami, où, voudrais-je penser, le spectacle de la vie urbaine et d'habitants unidimensionnels contribua à préparer l'émergence de l'idée directrice $^{4}$ ? Dans ces confessions rapides en tout cas, Williams montre à la fois que son penser procéda de sa propre résistance à la liquidation continue de la réserve. Dix ans après la rédaction de l'essai de Williams, Miami Vice rendait sensible une coïncidence de la coïncidence en participant à la résurrection de South Beach, comme en offrant l'ostension de

2. Comme, dans les années cinquante et soixante, le meilleur de: Alfred Hitchcock Presents, The Prisoner, The Twilight Zone, The Avengers, ou The Outer Limits.

3. Ce serait le signe de la monoforme de vie, pour jouer sur la catégorie inventée par Peter Watkins (se reporter au site Internet à l'adresse http://pwatkins.mnsi.net).

4. Raymond Williams, Television. Technology and Cultural Form, New York, Schocken Books, 1975, p. 145 . 


\section{Un sens de la réserve}

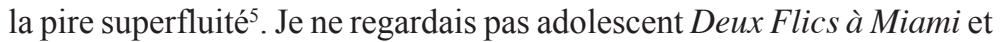
suis incapable aujourd'hui de visionner plus de quelques minutes d'un épisode. Il n'y a, voyez-vous, plus rien à voir quand tout est vu d'avance. L'à-plat des couleurs, la nullité de la « production » participent de cette grande entreprise de formatage. D'un côté, ce n'est assurément que de la télé, personne ne s'y tromperait (sauf les fous qui encenseront le film dit de cinéma, signé par Michael Mann, dans la suite de la série qu'il co-inventa). De l'autre, c'est une proposition, qui vous susurre cette forme pauvre, fais-en ta vie, et se réalise en plus d'une ou d'un. Miami Vice est donc l'apogée de la série-télé, réitérable éventuellement, mais indépassable à ce niveau. Le cran d'après dans la même direction fut la télé-réalité ${ }^{6}$, ou la vérification de la réduction performative, et hors des conventions de la série.

La real $T V$ a délivré nombre de séries de leur mission absolue, et, avec la relégation progressive des chiffres d'audience, a cristallisé l'apparition de la plupart des feuilletons qui sont commentées dans ces pages. Il est intéressant que Dexter, série policière des années 2000 située à Miami, en revienne à une production psychique moins effrayante que ce que déployait Miami Vice, et ce bien qu'elle démonte les processus mentaux d'un serial killer, à la fois protagoniste et narrateur. Dexter a peu d'épaisseur, il est du moins doté d'une voix intérieure. Le procédé résurgent de la voix off, s'est répandu de nouveau à partir de séries comme Desperate Housewives, et signale un possible décalage dans la formation du je. Mais pour l'essentiel, dès après l'épisode pilote, Dexter est surtout le parangon d'une double vie. Or chacune de ces deux formes d'existence tient dans la manifestation sans (trop de) reste: le charmant jeune homme, l'assassin méthodique. L'insistance sur l'absence de sentiments fait mettre en doute que la résistance soit plus que l'unité du corps et le code enseigné par le père adoptif. La vie secrète n'est donc pas tant un double fond qu'une duplication d'un seul système anthropogénique. En cela, Dexter se conforme à la terrifiante réponse que donna un jour Charles Manson au reporter de télévision qui lui demandait qui êtes-vous? Faisant des mimiques contradictoires, sourire, peur, surprise, incompréhension, Manson, qui déployait ainsi son répertoire visuel de manifestations affectives immotivées, ensuite murmura: «personne, je

5. Superfluité, n. c., caractère conjoint du superflu et du super-flux.

6. Là-dessus, relire Stéphane Breton, Télévision, Paris, Grasset, 2005. 
ne suis personne ». Exactement. Le Monsieur Tout le monde de cette mécanique psychique n'est personne de particulier, il n'est personne tout court, puisque la solidité du sujet s'est effondrée depuis beau temps, puis que la singularité s'évanouit, sous les effets multiplicateurs d'un enrégimentement télévisuel donné.

C'est alors à ce devenir vide que l'œuvre des séries saura s'opposer, et aujourd'hui plus particulièrement, où la métaphore de Miami, de la Californie, de l'américanisation ne devrait plus tromper sur la réalité transcontinentale d'un phénomène politique et mental. La proximité dans le personnel, les lieux de production, la grammaire artistique de base ne permettent nullement de poser sur un plan unique les « séries d'aujourd'hui », fût-ce un canon privilégié (HBO, ou autres). Les ressemblances font saillir des écarts immenses. Ainsi, la double vie de Donald Draper dans Mad Men n'est pas celle de Dexter. À travers les multiples avatars qui sont les siens, Don - incessamment pris, de toute façon, dans plus de deux destinées parallèles - incarne précisément la nécessité d'une résistance psychique, qui ne se réduit jamais ni à ni dans la manifestation. La majorité des autres personnages fonctionnent de la sorte, à part, évidemment, Betty Draper, la femme-enfant au foyer, qui est justement sortie des romans de Cheever ${ }^{7}$. Beaucoup de spectateurs, surtout dans le milieu des intellectuels et créateurs de gauche (qui, aux États-Unis, constitue l'écrasante proportion du public de Mad Men) éprouvent une honte rentrée lorsqu' ils sentent monter en eux une nostalgie pour le monde de Draper \& Co : comment être même fantasmatiquement solidaires d'un passé de confrontation, de conservatisme, qui opprime les minorités raciales et sexuelles, qui demeure phallocentrique? Cette nostalgie, à mon avis, découle plus de l'évidence présentée d'une réserve subjective, qui aurait donc eu cours dans la société de naguère, et qui est dépeinte au moment du processus historique menant à sa plus grande raréfaction. L'accélération de ce processus s'accorde sans hasard avec l'expansion télévisuelle, escortant la part dite glorieuse des civilisations méta-européennes ${ }^{8}$. Une série télévisée, en creux, et par un retour arrière, s'interroge ainsi sur la constitution de ce que permit un usage majeur

7. Rapprochement d'ailleurs suggéré par Natasha Vargas-Cooper, Mad Men Unbuttoned, New York, Collins Design, 2010, p. 159 sq.

8. On passerait aux États-Unis, entre 1950 et 1959 (année figurée dans la première saison de Mad Men), d'un peu moins de dix millions de téléviseurs à plus de 67 millions de postes. 
du medium, et qui est autrement amplifié désormais par la destruction quotidienne nommée facebook, etc. Voilà qui fait œuvre, et plus que des pans entiers de la littérature contemporaine, qui, eux, prennent acte de ces formes réduites, et ne cherchent plus qu'à leur donner la réplique, sans ouvrir d'espoir de contestation plus radicale. Paradoxalement, un passéisme de la tendance (les sixties de la série la plus hype, le désuet art déco dans la ville de nouveau trendy, le vintage du dandy 2.0) nous ferait-il trouver quelques moyens de nous mettre en réserve de l'unitaire manifestation du monde? 